\title{
Studies on Isotope Shift Exponent and Pressure Coefficient for Extended Superconductors within BCS Formalism
}

\author{
B. BANERJEE AND S.K. ROY* \\ Department of Physics, Visva-Bharati University, Santiniketan — 731 235, India
}

(Received March 5, 2008; revised version September 2, 2008)

\begin{abstract}
A tight-binding model comprising a hopping and an attractive interaction term was considered for high temperature superconductors. Explicit calculations on the effects of isotopic substitution of atoms and application of pressure on the transition temperatures were made in this work. Exact analytical expressions for the isotope-shift exponent $(\alpha)$ and pressure coefficient $\left(\gamma_{P}\right)$ of transition temperature $\left(T_{\mathrm{C}}\right)$ considering the isotopic mass and pressure dependence of the hopping and attractive interaction strengths from a minimal model describing superconductivity were obtained. Theoretical predictions for $\alpha$ and $\gamma_{P}$ with proper choice of parameters are found to be qualitatively consistent with the results of the experiments of high $T_{\mathrm{C}}$ oxides. The results depend on the band dispersion chosen.
\end{abstract}

PACS numbers: $74.20+\mathrm{f}, 74.25 . \mathrm{Bt}, 74.25 . \mathrm{Ha}$

\section{Introduction and motivation}

Since the discovery of superconductivity, the isotopic substitution $(\alpha)$ of atoms and the pressure dependence $\left(\gamma_{P}\right)$ of transition temperature have been recognised as two key factors [1-6] affecting the transition temperature $\left(T_{\mathrm{C}}\right)$. The isotope effects in superconductivity were taken as evidence for phonon mediation. In the lower $T_{\mathrm{C}}$ materials, for example $\mathrm{La}_{2-x} \mathrm{Sr}_{x} \mathrm{CuO}_{4}$ systems, $T_{\mathrm{C}}$ can be derived by changing the carrier concentration. Therefore, it is possible to examine the relationship between $T_{\mathrm{C}}$ and $\alpha$ with this structure [5].

In general, the isotope shift exponent $\alpha$ is large for BCS phonon mediated superconductor (exceeding the canonical value of 0.5 , in some cases) in the underdoped region. In many cases, $\alpha$ is very small at optimum doping where $T_{\mathrm{C}}$ is maximum and increases in the overdoped region. The interest in phonon mediated superconductivity in cuprates has also been revived due to the discovery of rather large isotopic coefficients $(\alpha)$ in $\mathrm{La}_{2-x} \mathrm{Sr}_{x} \mathrm{CuO}_{4}$ near $x \approx 0.12$ and similar experiments on $\mathrm{YBa}_{2} \mathrm{Cu}_{3} \mathrm{O}_{7-8}$ with $\mathrm{Pr}$ and $\mathrm{Gd}$ substituted at the $\mathrm{Y}$ or $\mathrm{Ba}$ sites as observed by Franck et al. [6]. Initial studies on isotope effects in high $T_{\mathrm{C}}$ superconductors found that the effect is either absent or very small $[7,8]$. Several experiments have been performed for high temperature superconductors for isotopic mass $[9,10]$ and pressure dependence [11-13].

Since the majority of these systems become superconducting on doping with holes, the dopant dependent isotope-shift exponent of $T_{\mathrm{C}}\left(\alpha=-\delta \ln T_{\mathrm{C}} / \delta \ln M\right)$

* corresponding author; e-mail: roysamar@redifmail.com and the pressure coefficient of the transition temperature $\left(\gamma_{P}=\delta \ln T_{\mathrm{C}} / \delta P\right)$ have been seriously studied over the years and certain general trends have been found [14, 22]. In the overdoped region, negative values of the pressure coefficients at high pressures and small negative values of $\alpha$ have been reported [9, 22]. For electron doped oxide systems, the pressure coefficient is negative and its value is large for low $T_{\mathrm{C}}$ samples and decreases with increasing $T_{\mathrm{C}}[11]$.

In the present work, we propose to study all the salient features of $\alpha$ and $\gamma_{P}$ with doping from our theoretical model by recasting the tight-binding Hamiltonian in the BCS form and apply a judicious choice of parameters to invoke the mass and pressure dependence of both the hopping and attractive interaction strengths.

\section{Theoretical model}

In our model, we begin with local pairing interaction in narrow-band systems with weak correlation. In the weak-correlation limit, the minimal relevant model for studying superconductivity would comprise of an attractive term for electrons. Thus our model Hamiltonian is

$$
H=-t \sum_{i, j, \sigma} a_{i \sigma}^{+} a_{j \sigma}-V \sum_{i, j} n_{i} n_{j}-\mu \sum_{i, \sigma} n_{i \sigma},
$$

where $t$ and $V$ are the hopping matrix element and effective attraction between electrons at nearest neighbour sites, respectively. $a_{i \sigma}^{+}\left(a_{i \sigma}\right)$ is the usual electron creation (annihilation) operator and $n_{i \sigma}\left(=a_{i \sigma}^{+} a_{i \sigma}\right)$ is the usual number operator corresponding to the $i$-th site and spin state $\sigma(=\uparrow \downarrow)$. Here $\mu$, the chemical potential, is determined by the filling of bands (doping in the case of oxide systems). 
Now we will consider the band dispersion including the next nearest neighbour given by $[15,16]$ :

$$
\begin{aligned}
\epsilon_{k} & =-2 t_{1}\left(\cos K_{x} a+\cos K_{y} a\right) \\
& +4 t_{2} \cos \left(K_{x} a\right) \cos \left(K_{y} a\right)-\mu .
\end{aligned}
$$

Here $\epsilon_{k}$ is the momentum dependent energy relative to the chemical potential $\mu, K$ is the wave vector and $t_{1}$ and $t_{2}$ are the nearest neighbour (NN) and next nearest neighbour (NNN) hopping terms.

As we are still in BCS approximation, the second term on the right hand side of Eq. (1) is simplified by mean-field approximation with introduction of an order parameter

$$
\Delta_{0}=\frac{1}{2}\left(\left\langle a_{i \downarrow} a_{j \uparrow}\right\rangle+\left\langle a_{j \downarrow} a_{i \uparrow}\right\rangle\right) .
$$

Then by performing momentum $k$-space transformation, the Hamiltonian takes the BCS form

$$
H=\sum_{k \sigma} \xi_{k} n_{k \sigma}+\sum_{k} \Delta_{k}\left(a_{k \uparrow}^{+} a_{-k \downarrow}^{+}+\text {h.c. }\right),
$$

where $\xi_{k}=\epsilon_{k}-\mu, \epsilon_{k}=-t z \gamma_{k}$ and $\gamma_{k}=$ $\left(\frac{1}{z}\right) \sum_{i j} \exp \left(\mathrm{i} \boldsymbol{k} \cdot \boldsymbol{R}_{i j}\right), R_{i j}$ is the nearest neighbour lattice vector and then the BCS type pairing, the superconducting gap parameter is given by

$$
\Delta_{k}=z \gamma_{k} \nu \Delta_{0}
$$

For a square lattice $z=4$ and $\gamma_{k}=\cos k_{x}+\cos k_{y}$.

\section{Evaluation of DOS and superconducting $T_{\mathrm{C}}$}

We now define the momentum dependent double time temperature dependent Green functions:

$$
\begin{aligned}
& G_{\bar{k} \bar{k}^{\prime}}(\omega)=\left\langle\left\langle a_{\bar{k} \uparrow} ; a_{\bar{k} \uparrow}^{+}\right\rangle\right\rangle, \\
& G_{k k^{\prime}}^{(1)}(\omega)=\left\langle\left\langle a_{-\bar{k}^{\prime} \downarrow}^{+} ; a_{\bar{k} \uparrow}^{+}\right\rangle\right\rangle .
\end{aligned}
$$

The density of states (DOS) near the Fermi surface is obtained by using Eqs. (6) and (7) and the transition temperature.

In general, DOS is defined by [14]:

$$
\rho(\omega)=-2 \sum_{\bar{k}} \operatorname{Im}\left\langle G_{\bar{k}}(\omega)\right\rangle,
$$

where

$$
G_{\bar{k}}(\omega)=\sum_{\bar{k}^{\prime}} \underset{G}{G}(1)=\frac{1}{2 \pi}(\omega) \frac{\omega}{\left(\omega-\epsilon_{\bar{k}^{\prime}}\right)\left(\omega+\mathrm{i} \Delta_{1}\right)}
$$

is the normal Green function at low temperature.

We consider the change in DOS from its unperturbed value to determine the effect on DOS due to alloying, thus we have

$$
\delta \rho(\omega)=-2 \sum_{\bar{k}} \operatorname{Im}\left(\left\langle G_{\bar{k}}(\omega)\right\rangle-G_{\bar{k}}^{\mathrm{ON}}(\omega)\right),
$$

where

$$
\begin{aligned}
G_{\bar{k}}^{\mathrm{ON}} \omega & =\lim _{\Delta \rightarrow 0} G_{\bar{k}}^{(0)} \omega, \\
\delta \rho(\omega) & =-\frac{1}{\pi} \sum_{\bar{k}} \operatorname{Im} \frac{\sum(\omega)}{\left(\omega-\epsilon_{\bar{k}}\right)^{2}},
\end{aligned}
$$

$$
\delta \rho(\omega)=\frac{\rho_{0}(\epsilon)}{\pi} \operatorname{Im} \sum(\omega) \frac{\delta}{\delta \omega} F(\omega),
$$

where $\sum(\omega)$ is the self-energy of the system.

At this stage, one needs to make a choice of $\rho_{0}(\epsilon)$. Under the simplest assumption of square DOS, $\rho_{0}(\epsilon)$ which becomes independent of $\epsilon, F(\omega)$ is constant and there is no change in DOS. Therefore, more general approximation could be to assume a Lorentzian form given by

$$
\begin{aligned}
& \rho_{0}(\epsilon)=\rho_{0}(0) \frac{D^{2}}{D^{2}+\epsilon^{2}}, \\
& F(\omega)=\pi \rho_{0}(0) \frac{D}{\omega+\mathrm{i} D},
\end{aligned}
$$

where $D$ is the band width. For square DOS, $D$ is large. $\epsilon_{\bar{k}}$ is the energy just above the Fermi surface.

The transition temperature $T_{\mathrm{C}}$ is obtained from the gap equation

$$
\Delta=-V \sum_{\bar{k}}\left\langle a_{\bar{k} \uparrow}^{+} a_{\bar{k} \downarrow}^{+}\right\rangle=-V \sum_{k}\left\langle a_{k \uparrow} a_{-k \downarrow}\right\rangle,
$$

where the gap correlation function is

$$
\begin{aligned}
& \left\langle a_{\bar{k} \uparrow}^{+} a_{\bar{k} \downarrow}^{+}\right\rangle=\mathrm{i} \int_{-\infty}^{\infty} \mathrm{d} \omega \\
& \quad \times \frac{\left[G^{(1)}(\omega+\mathrm{i} \epsilon)-G^{(1)}(\omega-\mathrm{i} \epsilon)\right]}{\mathrm{e}^{\beta \omega}+1} .
\end{aligned}
$$

Thus using Eqs. (6), (7) and (16), we obtain

$$
\left\langle a_{k \uparrow}^{+} a_{-k \downarrow}^{+}\right\rangle=-\frac{\Delta_{k}}{2 E_{k}} \tanh \left(\frac{\beta E_{k}}{2}\right)
$$

with $\beta=\frac{1}{k_{\beta} T}$ and $E_{k}=\sqrt{\xi_{k}^{2}+\Delta_{k}^{2}}$.

¿From the set of coupled Eq. (5) and Eq. (17) for the order parameters, one obtains at $T=T_{\mathrm{C}}$, the self-consistent equation for the gap as

$$
\Delta_{k}=-z \gamma_{k} V \sum_{r} \gamma_{r}\left[-\frac{\Delta_{r}}{2 E_{r}} \tanh \left(\beta E_{r / 2}\right)\right],
$$

which gives the equation for $T_{\mathrm{C}}$ as

$$
\begin{aligned}
1 & =z V \int_{-1}^{+1} \mathrm{~d} \epsilon_{r} N\left(\epsilon_{r}\right) \frac{\epsilon_{r}^{2}}{2\left(\epsilon_{r}-\mu_{r}\right)} \\
& \times \tanh \left(\beta_{\mathrm{c}}^{r} \frac{\epsilon_{r}-\mu_{r}}{2}\right) .
\end{aligned}
$$

Here $\epsilon_{r}, \mu_{r}, \beta_{\mathrm{c}}^{r}$ are a set of reduced variables, namely energy, chemical potential and $\frac{1}{k_{\beta} T_{\mathrm{C}}}$ in units of electronic half bandwidth $(W=4 t)$; i.e., $\epsilon_{r}=\frac{\epsilon}{W}, \mu_{r}=\frac{\mu}{W}$ and $\beta_{\mathrm{c}}^{r}=W \beta_{\mathrm{c}}$ with $\beta_{\mathrm{c}}=\frac{1}{k_{\beta} T_{\mathrm{C}}}$. The reduced electronic DOS $\left[\mathrm{N}\left(\epsilon_{\mathrm{r}}\right)\right]$ may be written in terms of electronic DOS, $N(\epsilon)$ as $N\left(\epsilon_{r}\right)=W N(\epsilon)$

\section{Isotope-shift exponent and pressure coefficient of $T_{\mathrm{C}}$}

The isotope-shift exponent $(\alpha)$ and the pressure coefficient $\left(\gamma_{P}\right)$ of $T_{\mathrm{C}}$ is defined as $\alpha=-\frac{\partial \ln T_{\mathrm{C}}}{\partial \ln M}$ and $\gamma_{P}=\frac{\partial \ln T_{\mathrm{C}}}{\partial P}$.

Hence to derive $\alpha$ and $\gamma_{P}$ we need to differentiate Eq. (19) with respect to $M$ and $P$. The basic parameters in the model which govern $T_{\mathrm{C}}$ are the hopping and attractive pairing strengths. In keeping with the experimental 
evidence of a change in the effective mass of charge carriers with isotopic substitution in $\mathrm{Y} 123\left(\mathrm{YBa}_{2} \mathrm{Cu}_{3} \mathrm{O}_{7-\text { delta }}\right.$ samples [17], $M$ dependence of $t$ is fairly obvious. But till date, there is no real experimental estimate for the $M$ dependence of $V$. However, it may be assumed that $V$ is mass dependent through its dependence on $t$ (in analogy with the $t-J$ model). Thus the expression for $\alpha$ will be

$$
\alpha=-\frac{\partial \ln t}{\partial \ln M}+\left(\frac{\partial \ln t}{\partial \ln M}-\frac{\partial \ln V}{\partial \ln M}\right) \frac{1}{\beta_{\mathrm{c}}^{r} z V P_{1}} .
$$

As the pressure reduces lattice spacing, it is bound to assist hopping and the strength of the attractive interaction also should grow with applied pressure. The application of pressure will alter the carrier concentration in high $T_{\mathrm{C}}$ oxide systems. Thus the expression for $\gamma_{P}$ may be written as

$$
\begin{aligned}
\gamma_{P} & =\frac{\partial \ln t}{\partial P}+\left(-\frac{\partial \ln V}{\partial P}-\frac{\partial \ln t}{\partial P}\right) \frac{1}{\beta_{\mathrm{c}}^{r} z V P_{1}} \\
& +\left(\frac{P_{2}}{\beta_{\mathrm{c}}^{r}}-\frac{P_{3}}{2}\right) \frac{1}{P_{1}} \frac{\partial \mu_{r}}{\partial P},
\end{aligned}
$$

where

$$
\begin{aligned}
P_{1} & =\int_{-1}^{+1} \mathrm{~d} \epsilon_{r} N\left(\epsilon_{r}\right) \frac{\epsilon_{r}^{2}}{4} \operatorname{sech}^{2}\left(\beta_{\mathrm{c}}^{r} \frac{\epsilon_{r}-\mu_{r}}{2}\right), \\
P_{2} & =\int_{-1}^{+1} \mathrm{~d} \epsilon_{r} N\left(\epsilon_{r}\right) \frac{1}{2}\left(\frac{\epsilon_{r}}{\epsilon_{r}-\mu_{r}}\right)^{2} \\
& \times \tanh \left(\beta_{\mathrm{c}}^{r} \frac{\epsilon_{r}-\mu_{r}}{2}\right),
\end{aligned}
$$

and

$$
\begin{aligned}
P_{3} & =\int_{-1}^{+1} \mathrm{~d} \epsilon_{r} N\left(\epsilon_{r}\right) \frac{1}{2}\left(\frac{\epsilon_{r}^{2}}{\epsilon_{r}-\mu_{r}}\right) \\
& \times \operatorname{sech}^{2}\left(\beta_{\mathrm{c}}^{r} \frac{\epsilon_{r}-\mu_{r}}{2}\right) .
\end{aligned}
$$

The term $\frac{\partial \mu_{r}}{\partial P}$ occurring in the expression for the pressure coefficient i.e., in Eq. (21) may be written in terms of the change in the carrier concentration $(n)$ as

$$
\frac{\partial \mu_{r}}{\partial P}=\frac{1}{2 N\left(\mu_{r}\right)} \frac{\partial n}{\partial P} .
$$

The isotope shift exponent $(\alpha)$ and the transition temperature $\left(T_{\mathrm{C}}\right)$ are calculated using a square DOS and DOS with a van Hove singularity (vHs) [18, 19]. From Eq. (25) it is shown than for a square DOS

$$
\frac{\partial \mu_{r}}{\partial P}=\frac{\partial n}{\partial P}
$$

as for a square (energy-independent DOS),

$$
N\left(\epsilon_{r}\right)=\frac{1}{2}, \quad\left|\epsilon_{r}\right| \leq 1 .
$$

For a normalised DOS with a vHs [18],

$$
\frac{\partial \mu_{r}}{\partial P}=0.20953 \ln \left|\frac{4}{\epsilon_{r}}\right| \frac{\partial n}{\partial P} .
$$

The role of second nearest neighbour hopping in hole-doped high- $T_{\mathrm{C}}$ cuprates are important. For calculating the isotope shift exponent $\alpha$, we will reform the expression for $P_{1}$ from integration to summation form as given by

$$
I_{1}=\frac{1}{N} \sum_{k}\left(\frac{\epsilon_{k}}{2 W}\right)^{2} \operatorname{sech}^{2}\left[\beta^{\mathrm{c}}\left(\frac{\epsilon_{k}-\mu_{r}}{2}\right)\right] .
$$

Thus using Eqs. (20), (29) and (2) the isotope shift exponent $\alpha$ can be calculated and the pressure coefficient $\left(\gamma_{P}\right)$ can also be evaluated in a similar way from Eq. (21) and the dispersion (Eq. (2)) using integrals $P_{1}, P_{2}$ and $P_{3}$ numerically.

\section{Numerical results}

The isotope shift exponent $(\alpha)$ and pressure coefficient $\left(\gamma_{P}\right)$ for BCS type superconductors and the relevant theories applied to high temperature superconductors (HTSC) compounds like Y123 and lanthanum strontium cobalt oxide (LSCO) compounds have been numerically evaluated for $s$-wave dispersion relation (2). The results are given in Figs. 1-6. Figure 1 represents the variation of transition temperatures $\left(T_{\mathrm{C}}\right)$ with chemical potential $\mu_{r}$ in terms of reduced variable with potentials mentioned in the figure caption of Fig. 1.

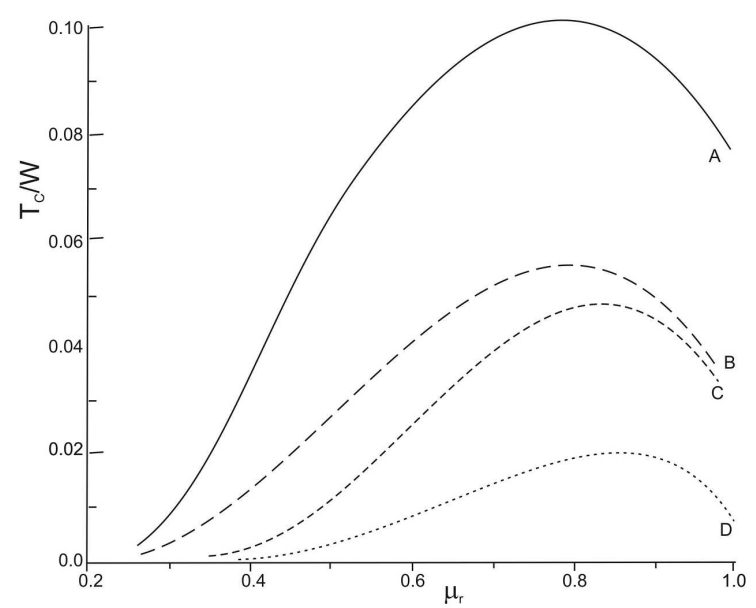

Fig. 1. Variation of $T_{\mathrm{C}}$ with $\mu_{r}$. Curves $A$ and $C$ are for $V_{r}=0.628$ and 0.4 , respectively, and a square DOS while $B$ and $D$ are for the same set of $V_{r}$ values with vHs in DOS.

The variations of $\alpha$ with $\mu_{r}$ are given in Figs. 2 and 3 and the variation of $\alpha$ with $T_{\mathrm{C}}$ is given in Fig. 4 for various values of $V_{r}, T_{\mathrm{C}}$ and other related parameters.

In a similar way the variation of pressure coefficients $\left(\gamma_{P}\right)$ with $T_{\mathrm{C}}$ and $\mu_{r}$ have been plotted in Figs. 5 and 6 , respectively, with $V_{r}=0.628$ and also using other related parameters. The figures are superimposed with experimental data for both hole doped Y123 systems and electron doped materials.

\section{Discussions}

In the present work we highlight the study of $\alpha, \gamma_{P}$ and $T_{\mathrm{C}}$ as a function of doping (through $\mu_{r}$ ) and discuss the qualitative comparisons of their variations with the general trends emerging from experiments on various high 


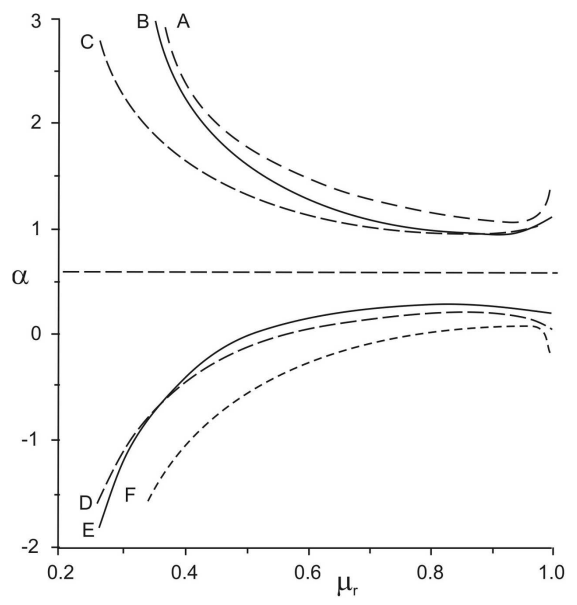

Fig. 2. Variation of $\alpha$ vs. $\mu_{r}$ with $(\delta \ln t) /(\delta \ln M)=$ -0.55 . Curves $A, B$ and $C$ are for $(\delta \ln V) /(\delta \ln M)=$ -0.75 while $D, E$ and $F$ are for $\delta \ln V / \delta \ln M=-0.45$. Other parameters for the curves are as follows: curves $A$ and $F: V_{r}=0.42$, vHs in DOS; curve $B: V_{r}=0.42$, square DOS; curves $C$ and $E: V_{r}=0.628$, vHs in DOS and curve $D: V_{r}=0.628$ and a square DOS.

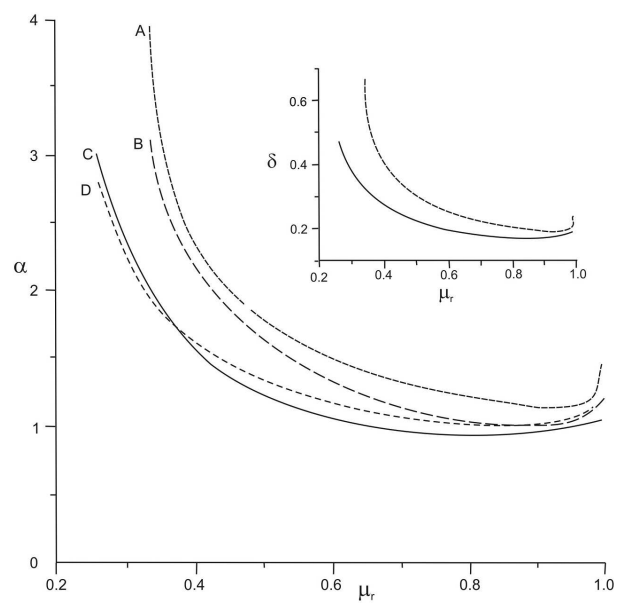

Fig. 3. $\alpha$ vs. $\mu_{r}$ with $\delta \ln t / \delta \ln M=-0.55$ and $\delta \ln V / \delta \ln M=-0.75$. Curves $B$ and $C$ are for a square DOS and $V_{r}=0.45$ and 0.628 , respectively, while curves $A$ and $D$ are for a vHs in DOS for the same set of $V_{r}$ values. The solid and the dotted curves in the inset are for $V_{r}=0.628$ and 0.42 , respectively, and a $\mathrm{vHs}$ in the DOS; $\delta \ln t / \delta \ln M$ and $\delta \ln V / \delta \ln M$ are scaled down to values of -0.11 and -0.133 , respectively, to obtain realistic value of $\alpha$.

$T_{\mathrm{C}}$ cuprates. Obviously, a suitable choice of parameters such as $V_{r}$, the mass and pressure dependence of $t$ and $V$ and the pressure dependence of carrier concentration (n) are made from Ref. [19]. The choice of parameters like $(\partial \ln t) / \partial \ln M$ is based on experimental results [17] on Y123 compounds, where $\left(\partial \ln m^{*}\right) / \partial \ln M_{0}$ has been reported to be $\approx 0.6 \mathrm{~m}^{*}$, where $\mathrm{m}^{*}$ and $M_{0}$ being the effective mass of the charge carrier and average mass of the oxygen atom, respectively. The effective mass of the

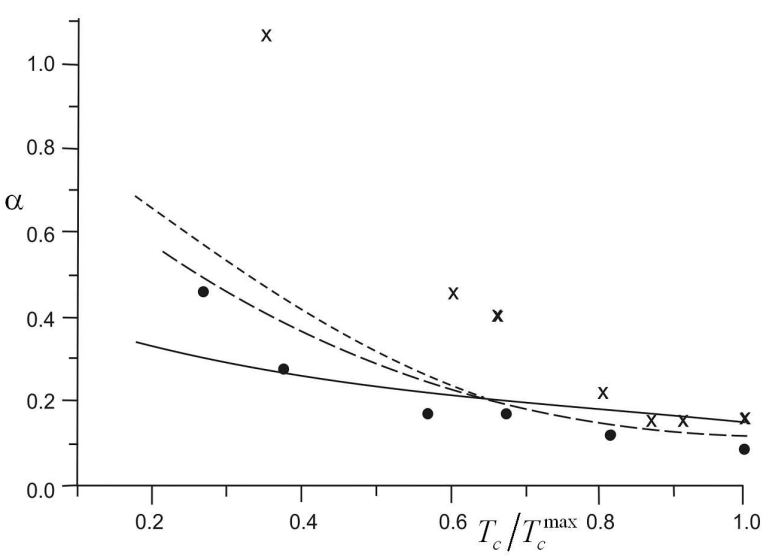

Fig. 4. $\alpha$ vs. $T_{\mathrm{C}} / T_{\mathrm{C}}^{\max }$ with $\delta \ln t / \delta \ln M=-0.052$ and $\delta \ln V / \delta \ln M=-0.1$. The solid curve is for $r_{2}=0$ (only NN hopping) while the dashed curves are for $r_{2}=0.81$ (longer dash) and 0.91 (shorter dash). The crosses and full circles denote the data for $\mathrm{La}^{214}$ and Co-substituted Y123 systems, taken from Ref. [14].

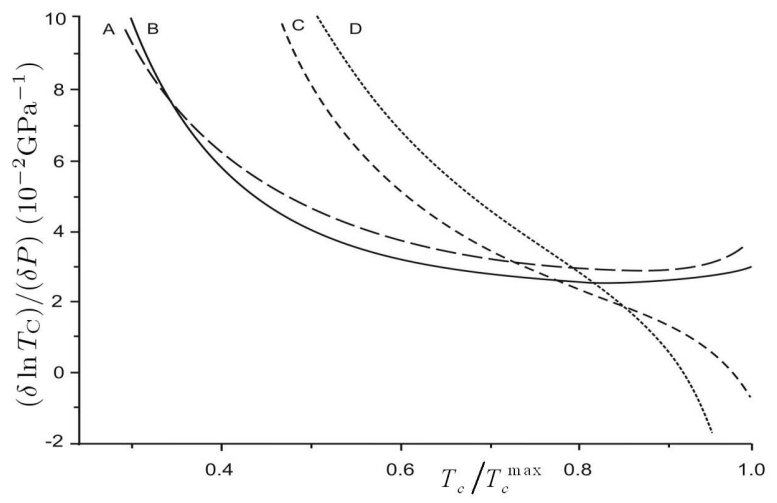

Fig. 5. $\delta \ln T_{\mathrm{C}} / \delta P$ in units of $10^{-2} \mathrm{GPa}^{-1}$ vs. $\mu_{r}$ with $\delta \ln t / \delta P=1 \times 10^{-2} \mathrm{GPa}^{-1}, \delta \ln V / \delta P=$ $2 \times 10^{-2} \mathrm{GPa}^{-1}$, and $V_{r}=0.628$. Curves $A$ and $B$ are for $\delta \mu_{r} / \delta P=0$ while $C$ and $D$ are for $\delta \mu_{r} / \delta P=$ $1 \times 10^{-2} \mathrm{GPa}^{-1}$. Curves $A$ and $C$ are for a square DOS while $B$ and $D$ are a vHs in DOS.

charge carrier is inversely proportional to the effective hopping strength, we treat $(\partial \ln V) / \partial \ln M$ as a free parameter.

In Fig. 1, we have shown the variation of $T_{\mathrm{C}} / W$ with $\mu_{r}$ for different values of $V_{r}$ and for two different types of DOS (square DOS and vHs DOS).

In Figs. 2 and $3 \alpha$ is plotted with $\mu_{r}$. It is found that $\alpha$ depends on the choice of $(\partial \ln V) / \partial \ln M$, the nature of DOS and $V_{r}$ for a given value of $(\partial \ln t) / \partial \ln M$. In our earlier work [19], we have seen that if only the hopping strength depends on the isotope mass, then $\alpha<0$ for all $\mu_{r}$, which corresponds to setting $(\partial \ln V) / \partial \ln M=0$. We choose $(\partial \ln V) / \partial \ln M$ as -0.45 and -0.75 .

Numerical exercises confirm that $P_{1}$ in Eq. (22) is positive definite for all $\mu_{r}$ values and three distinct features 


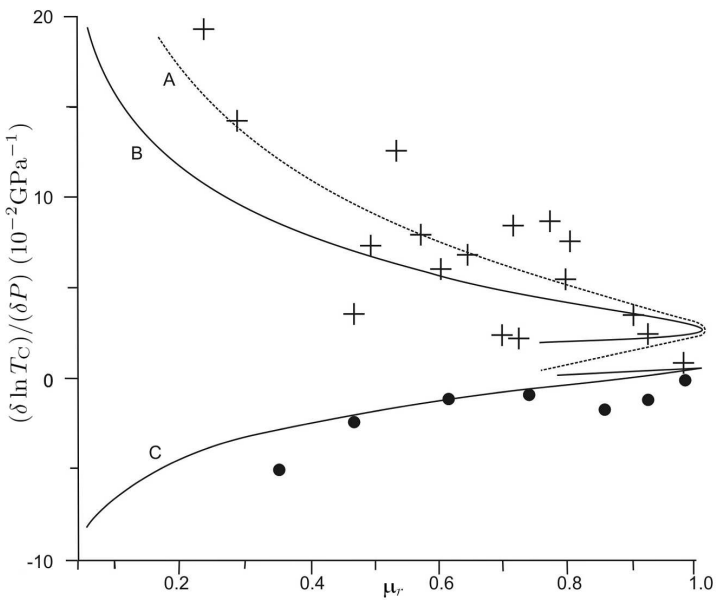

Fig. 6. $\delta \ln T_{\mathrm{C}} / \delta P$ vs. $T_{\mathrm{C}} / T_{\mathrm{C}}^{\max }$ for $V_{r}=0.628$ and a square DOS. Values of $\delta \ln t / \delta P$ and $\delta \ln V / \delta P$ are the same as the ones used in Fig. 5. For curves $A$ and $B, \delta \mu_{r} / \delta P=0.3$ and 0.7 , respectively, in units of $10^{-2} \mathrm{GPa}^{-1}$. For curve $C, \delta \ln t / \delta P, \delta \ln V / \delta P$ and $\delta \mu_{r} / \delta P$ are chosen as 2,1 and 0 in units of $10^{-2} \mathrm{GPa}^{-1}$. Superposed on the graphs are experimental data for both hole-doped (Y123 system represented by + sign) and electron-doped materials $\left(\mathrm{Nd}_{2-x} \mathrm{Ce}_{x} \mathrm{CuO}_{4}\right.$ system represented by full circles) taken from Ref. [22].

of $\alpha$ depending on the relative values of $(\partial \ln V) / \partial \ln M$ and $(\partial \ln t) / \partial \ln M$ emerge.

i) For $(\partial \ln V) / \partial \ln M>(\partial \ln t) / \partial \ln M, \alpha$ increases from large negative values for low values of $\mu_{r}$ to small positive values as $\mu_{r}$ increases.

ii) For $\frac{\partial \ln V}{\partial \ln M}<\frac{\partial \ln t}{\partial \ln M}$, $\alpha$ decreases from large positive values when $T_{\mathrm{C}}$ is low to a near minimum at optimum doping and increases slightly on the overdoped side.

iii) For $(\partial \ln V) / \partial \ln M=(\partial \ln t) / \partial \ln M$, equation yields $\alpha=-(\partial \ln t) / \partial \ln M$, which is a constant and independent of the nature of DOS and doping $\left(\mu_{r}\right)$.

It is to be noted that in Fig. 2 the dashed line corresponds to the case where $\alpha$ is constant and independent of doping, similar to BCS case. The other curves $(A, B$, $C, D, E$, and $F$ ) are given in the figure caption.

The dependence of $\alpha$ on $V_{r}$ and from the nature of DOS, we plot $\alpha$ against $\mu_{r}$ for $V=0.42$ and 0.628 and for both types of DOS (square and $\mathrm{vHs}$ ) structures, holding $(\partial \ln V) / \partial \ln M$ and $(\partial \ln t) / \partial \ln M$ fixed at -0.75 and -0.55 , respectively, in Fig. 3.

The effect of second NN hopping (through the dimensionless parameter $r_{2}=2 t_{2} / t$ ) on the behaviour of $\alpha$ with experimental data for $\mathrm{La}(214)$ and co-substituted Y123 systems have been shown in Fig. 4 for a comparison. The values of $r_{2}=0.81$ and 0.91 have been taken from earlier work [17]. The inclusion of NNN hopping enhances $\alpha$ considerably for small values of $T_{\mathrm{C}}$ and supresses $\alpha$ slightly near $T_{\mathrm{C}}$ of the order of $T_{\mathrm{C}}^{\max }$.

The variation of pressure coefficient $\gamma_{P}$ with $\mu_{r}$ for different values of $\partial \mu_{r} / \partial P$ but fixed values of $(\partial \ln t) / \partial P$ and $(\partial \ln V) / \partial P$ have been shown in Fig. 5 . We choose $(\partial \ln V) / \partial P>(\partial \ln t) / \partial P$ for the entire range of $\mu_{r}$ (for $\gamma_{P}$ positive) provided $\left(\partial \mu_{r}\right) / \partial P$ is small. $\gamma_{P}$ is large for low values of $\mu_{r}$, where $T_{\mathrm{C}}$ is small and decreases as $\mu_{r}$ increases.

In Fig. 6, we show the variation of $T_{\mathrm{C}}$ with $T_{\mathrm{C}} / T_{\mathrm{C}}^{\max }$ for two sets of values of $(\partial \ln V) / \partial P$ and $(\partial \ln t) / \partial P$, where $T_{\mathrm{C}}^{\max }$ is the transition temperature at optimum doping. For $(\partial \ln V) / \partial P>(\partial \ln t) / \partial P$, the pressure coefficient is large and positive for underdoped systems for low $T_{\mathrm{C}}$ values, whereas for $(\partial \ln V) / \partial P<(\partial \ln t) / \partial P$, the pressure coefficient is negative for underdoped systems. In both cases, the magnitude of pressure coefficients decreases with increasing $T_{\mathrm{C}}$ up to optimum doping.

\section{Conclusions}

¿From Figs. 1 to 6, one may conclude that for a simple minimal model considered in the present case, the variation of $\alpha$ and $\gamma_{P}$ with $T_{\mathrm{C}}$ for both electron doped and hole doped oxide superconductors may be explained even by recasting the Hamiltonian in the BCS form. We should mention here one of the limitations of our study. We have considered only the case of extended $s$-wave pairing. However, large number of evidences are present in favour of $d$-wave pairing in high $T_{\mathrm{C}}$ oxide systems which is supposed to modify the results $[20,21]$, the anisotropic extended $s$-wave pairing cannot be ruled out. We justify by saying that the present model is a very good approximation as the results agree well with the experimental values.

In brief, we summarise our results by stating that the exact analytical expressions for isotope shift exponent and the pressure coefficient of $T_{\mathrm{C}}$ considering the isotopic mass and pressure dependence of hopping and attractive interaction strength can be derived for a minimal model system describing an oxide superconductor.

\section{Acknowledgments}

Authors are highly thankful to Dr Sourabh Banerjee, Durgapur Govt. College, for his suggestions and related help.

\section{References}

[1] G.M. Zhao, M.B. Hunt, H. Keller, K.A. Müller, Nature 385, 236 (1997).

[2] R. Khasanov et al., Phys. Rev. Lett. 92, 057602 (2004).

[3] G.H. Gweon et al., Nature 430, 187 (2004).

[4] A. Bussman-Holder, H. Keller, Eur. Phys. J. B 44 , 487 (2005). 
[5] M.K. Crawford, M. Kunchur, W.E. Farneth, E.M. McCarron, S.J. Poon, Phys. Rev. B 41, 182 (1990).

[6] J.P. Franck, J. Jung, M.A.K. Mohamed, S. Gygax, G. Sproule, Phys. Rev. B 44, 5318 (1991).

[7] L.C. Bourne et al., Phys. Rev. Lett. 58, 2337 (1987).

[8] B. Batlog et al., Phys. Rev. Lett. 58, 2333 (1987).

[9] H.J. Bornemann, D.E. Morris, H.B. Liu, Physica $C$ 182, 132 (1991).

[10] H.J. Bornemann, D.E. Morris, H.B. Liu, P.K. Narwankar, Physica $C$ 191, 211 (1992).

[11] J.T. Markert, J. Beille, J.J. Neumeier, E.A. Early, C.L. Seaman, T. Moran, M.B. Maple, Phys. Rev. Lett. 64, 80 (1990).

[12] R. Griessen, Phys. Rev. B 36, 5284 (1987).

[13] C. Murayama, N. Mori, S. Yomo, H. Takagi, S. Uchida, Y. Tokura, Nature 339, 293 (1989).

[14] J.P. Franck, Physical Properties of High-Temperature Superconductivity, Ed. D.M. Ginsberg, Vol. 4, World Sci. Singapore 1994, p. 189.
[15] M. Abrecat et al., Phys. Rev. Lett. 91, 057002 (2003).

[16] A. Abrecat, D. Ariosa, D. Cloetta, S. Mitrovic, M. Omellion, X.X. Xi, G. Margaritondo, D. Pavuna, Phys. Rev. Lett. 91, 057002 (2003).

[17] Guo-meng Zhao, D.E. Morris, Phys. Rev. B 51, 16487 (1995).

[18] B. Banerjee, S.K. Roy, Int. J. Mod. Phys. B, 2008, submitted for publication.

[19] B. Banerjee, S.K. Roy, Indian J. Phys. 82, 257 (2008).

[20] Z.-X. Shen, D.S. Dessau, B.O. Wells, D.M. King, W.E. Spicer, A.J. Arko, D. Marshall, L.W. Lombardo, A. Kapitulnik, P. Dickinson, S. Doniach, J. DiCarlo, A.G. Loeser, C.H. Park, Phys. Rev. Lett. 70, 1553 (1993); Z.-X. Shen, W.E. Spicer, D.M. King, D.S. Dessau, B.O. Wells, Science 267, 343 (1995).

[21] C.C. Tsuei et al., Phys. Rev. Lett. 73, 593 (1994).

[22] J.S. Schilling, S. Klotz, Physical Properties of High-Temperature Superconductivity, Vol. 3, Ed. D.M. Ginsberg, World Sci., Singappore 1992, p. 59 . 